\title{
Puissance maternelle et pouvoir politique. La régence des reines mères
}

\section{Fanny Cosandey}

\section{(2) OpenEdition}

1 Journals

\section{Édition électronique}

URL : https://journals.openedition.org/clio/1447

DOI : $10.4000 /$ clio. 1447

ISSN : 1777-5299

Éditeur

Belin

\section{Édition imprimée}

Date de publication : 1 avril 2005

Pagination : 69-90

ISBN : 2-85816-781-8

ISSN : 1252-7017

\section{Référence électronique}

Fanny Cosandey, "Puissance maternelle et pouvoir politique. La régence des reines mères », Clio. Histoire, femmes et sociétés [En ligne], 21 | 2005, mis en ligne le 01 juin 2007, consulté le 22 avril 2022. URL : http://journals.openedition.org/clio/1447 ; DOI : https://doi.org/10.4000/clio.1447

Ce document a été généré automatiquement le 22 avril 2022.

Tous droits réservés 


\section{Puissance maternelle et pouvoir politique. La régence des reines mères}

Fanny Cosandey

1 La régence, sous l'Ancien Régime, va prioritairement aux femmes. S'il n'est pas possible d'affirmer qu'elle est automatiquement confiée aux reines mères, car celles-ci ne font jamais l'économie de manœuvres politiques pour s'assurer le pouvoir, force est de constater cependant qu'elles parviennent toujours à l'obtenir, au moins lors des minorités royales. Cette dévolution n'est pas purement institutionnelle. De fait, s'il était inscrit que les reines mères devaient statutairement assurer la régence, elles n'auraient nullement besoin de jouer des partis en présence pour confisquer l'autorité et opérer, de la sorte, ce qui peut être qualifié de coup de force politique ${ }^{1}$. Mais la qualité maternelle représente indéniablement un atout fort qui donne à la souveraine un avantage considérable sur ses compétiteurs, princes du sang ou grands dignitaires. Les propagandistes célébrant ce nouveau pouvoir, féminin, ne se font pas faute de le rappeler. Aux lois de l'État s'ajoute donc la loi de nature qui désigne la mère, plus que quiconque, pour s'occuper de l'éducation de son jeune fils, et défendre les intérêts de sa progéniture. À la fois reine, femme et mère, le personnage royal féminin présente de multiples facettes qui jouent tant dans le processus d'attribution de la régence que dans les conditions d'exercice de ce gouvernement d'exception.

2 Mais au-delà de la question du pouvoir féminin, c'est celle de la place des femmes dans la vie politique qui est alors soulevée. Car si la loi salique, qui réserve le trône aux mâles de la famille royale, est propre à la France, la régence au féminin, à l'inverse, est loin d'être une spécificité de cette monarchie. À considérer les autres royaumes chrétiens à la même époque, il apparaît que les femmes sont régulièrement mobilisées pour pallier les faiblesses d'un pouvoir masculin. Il n'est que d'évoquer le gouvernement des PaysBas sous les Habsbourg au XVI ${ }^{\mathrm{e}}$ siècle, la régence de Marie-Anne d'Autriche en Espagne pendant la minorité de Charles II, ou encore celle de la reine d'Écosse Marie de Guise pendant le bas âge de Marie Stuart, quelques exemples pris parmi tant d'autres, pour se 
convaincre du rôle essentiel que jouent les femmes dans les modalités de gestion et de transmission du pouvoir royal ${ }^{2}$.

3 D'une façon plus générale encore, la participation féminine aux affaires doit être replacée dans le cadre de la construction familiale: les fonctions de l'épouse, de la mère, de la veuve, dépassent largement le domaine restreint de l'espace domestique pour s'affirmer dans le monde, à travers des activités que l'on pourrait qualifier de " publiques » bien que le terme ne soit pas absolument clarifié dans la société d'Ancien Régime ${ }^{3}$. Les travaux récents sur l'histoire des femmes révèlent en effet des «lieux » d'intervention parfois inattendus ${ }^{4}$ et rétablissent ce faisant l'équilibre entre hommes et femmes dans le fonctionnement de la société tout entière. Cette perspective conduit à récuser la figure d'une régence féminine entendue comme le dérèglement provisoire d'une autorité masculine par essence.

4 L'intervention politique des femmes n'est d'ailleurs pas condamnée par les contemporains ${ }^{5}$, même s'il y a une contradiction forte, en France, entre la justification de la loi salique considérée comme une loi de nature écartant le sexe faible du pouvoir politique, et la célébration d'une régence féminine qui désigne, naturellement, les reines mères au gouvernement. En fait, les jurisconsultes prennent soin de traiter séparément ces deux questions d'ordre institutionnel qui ne répondent pas aux mêmes impératifs politiques mais renvoient au même problème : celui de la place des femmes dans l'État, dans la famille et, finalement, dans la constitution et la transmission du patrimoine, qu'il soit public ou privé. La relation entre la construction de l'appareil d'État et l'affirmation des familles qui le peuplaient a été mise en évidence à travers le concept de Family State compact développé par Sarah Hanley ${ }^{6}$. Le rôle joué par le droit privé dans l'élaboration théorique de la monarchie se rencontre encore dans les multiples emprunts faits au modèle familial ${ }^{7}$; la figure patriarcale du roi est même une composante de l'absolutisme français ${ }^{8}$. La régence ne fait pas l'économie de cette comparaison et la reine, mère du roi, peut apparaître comme une mère de la patrie par la place qu'elle occupe dans le gouvernement ${ }^{9}$. À l'instar du droit privé, l'épouse devenue veuve se voit confier la tutelle des enfants mineurs et, quand l'éducation rejoint la direction des affaires de l'État, obtient ainsi les rênes du pouvoir.

C'est cette articulation entre données institutionnelles et données sociales, entre pratiques politiques et pratiques familiales qu'il s'agit ici d'analyser, tant dans les discours tenus sur la régence que dans l'effectivité du pouvoir exercé par celle qui est, statutairement, exclue de l'héritage capétien sans être écartée de la scène politique. À la faveur qui la porte, en tant que femme, à gouverner lors d'une minorité royale, s'ajoute l'influence d'une mère sur un enfant en bas âge qui reste, quoi qu'il en soit, détenteur de l'autorité royale.

De la tutelle du prince au gouvernement du royaume

6 Une étude de la régence féminine ne peut faire l'économie de la structure institutionnelle dans laquelle elle s'inscrit. L'installation des femmes dans la régence est en fait, aussi paradoxal que cela puisse paraître, une conséquence directe de leur exclusion du trône. Plusieurs étapes les conduisent à assurer la continuité monarchique en cas de défaillance de l'autorité royale masculine. Nous les rappelons ici pour mémoire ${ }^{10}$.

7 Des crises successorales de 1316 et 1328 naissent les deux principes qui régissent la transmission du trône de France: les femmes ne peuvent hériter ni transmettre les droits à la couronne. La souveraineté, strictement masculine dans son exercice, est 
dévolue au plus prochain mâle en ligne masculine. La couronne s'affirme donc indisponible et le domaine royal devient, corrélativement, inaliénable. Pour compléter cette construction juridique, et assurer de la sorte la pérennité de la royauté maintenue dans une seule et même lignée dynastique, Charles V, puis son successeur Charles VI, fixent les conditions d'exercice de la régence en cas de minorité royale. Avec l'ordonnance de 1374 , les femmes se voient confier la tutelle du roi mineur, tandis que les princes et grands dignitaires du royaume ont la charge du gouvernement. Rien de très original à cela : dans la société féodale, les mères obtiennent traditionnellement la garde de leurs enfants jusqu'à la majorité. C'est sur ce modèle que se calque l'organisation de la régence dans un premier temps. Seule la majorité du roi, fixée à 14 ans par cette même ordonnance, met un terme au pouvoir du régent. La protection du roi mineur est assurée par la reine mère, mais les risques d'usurpation, par un prince trop ambitieux, sont loin d'être écartés. Pour pallier de tels dangers, Charles VI, par une ordonnance promulguée en 1407 , révise cette forme de gouvernement transitoire. En établissant le principe d'instantanéité de la succession - à l'origine d'un roi-qui-nemeurt-jamais - il modifie profondément la conception même de la régence, désormais exercée au nom d'un monarque qui, bien que mineur, détient seul l'intégralité de la souveraineté. Il n'est plus question, dès lors, que d'une délégation de l'autorité incarnée par le roi. Et la reine trouve ainsi place dans le gouvernement, passant d'une fonction essentiellement domestique à une fonction éminemment politique. En effet, le changement opéré dans la personne du roi conduit à reconsidérer l'exercice du pouvoir en associant celle qui s'occupe du souverain mineur et ceux qui, par leur naissance ou leurs hautes fonctions, sont légitimes dans la gestion des affaires de l'État. De là provient le caractère collégial de la régence, où la reine mère partage le pouvoir avec les princes du sang et les grands dignitaires. Mais ce gouvernement à têtes multiples représente un idéal qui est difficilement appliqué dans les faits, l'autorité se concentrant plus volontiers dans les mains d'un seul. Et c'est aux reines mères que revient finalement la responsabilité du gouvernement, celles-ci s'instituant, par touches successives, plus légitimes que d'autres dans ces fonctions si convoitées. Le modèle strictement féminin de la régence ne s'impose cependant qu'au terme d'un long processus.

8 Jusqu'en 1483, la France ne connaît pas de minorités royales ; pendant plus d'un demisiècle les dispositions prises par Charles VI concernant la régence demeurent donc lettre morte. Mais le principe d'instantanéité de la succession est désormais intégré au fonctionnement monarchique. À la mort de Louis XI, son successeur est encore un enfant de treize ans et le roi moribond confie à sa fille aînée, Anne de Beaujeu, et à son gendre, le soin des affaires et de l'éducation du jeune héritier. Si le premier prince du sang, Louis d'Orléans, dénonce la mainmise de la princesse royale sur le gouvernement, il n'obtient cependant pas son renvoi. Un précédent est né : celle qui tient le roi tient aussi, de fait, les rênes de l'État ${ }^{11}$. L'installation des femmes dans la régence se confirme par la suite. Gravement malade, Louis XII rédige son testament et propose d'associer la reine, Anne de Bretagne ${ }^{12}$, et la mère du futur François $1^{\text {er13 }}$, pour diriger le royaume le temps de la minorité royale. Deux femmes, donc, sont désignées à la tête de l'État ; deux statuts, aussi, se rejoignent ici : la reine Anne, détentrice de la plus haute dignité qui soit en terre de France, et la mère, Louise, dévouée aux intérêts de sa progéniture. Si cette régence n'eut pas lieu, Louis XII s'étant finalement rétabli, le souvenir en est resté et conforte la position des femmes à la tête du gouvernement lors d'une incapacité royale. D'autant plus qu'à la même époque, les reines de France se voient confortées 
dans la dignité souveraine, et partagent avec leurs époux tous les acquis de cette position d'exception, le pouvoir royal mis à part. Elles bénéficient ainsi de tous les signes extérieurs de la royauté, connaissent elles aussi les grandes cérémonies monarchiques qui marquent l'appartenance au trône (sacre, entrées, funérailles), incarnent comme le roi la grandeur souveraine. Incapables de recueillir l'héritage capétien, elles peuvent être dotées de tous les attributs de la souveraineté sans qu'il y ait de confusion quant à celui qui est source de pouvoir. Elles sont, dès lors, parfaitement intégrées à la monarchie à l'instant même où le sacrement du mariage leur communique la dignité de l'époux. Dans une telle perspective, le rôle de la reine en temps de régence se comprend aisément: femme, elle ne représente aucun risque d'usurpation; reine, elle bénéficie d'un statut hors du commun, première dame du royaume et second personnage après le roi. Si elle est mère de surcroît, elle peut se prévaloir de la confiance que lui confère le lien, naturel, qu'elle entretient avec le petit roi. Autant d'atouts qui la désignent au premier poste de commandement lorsque le roi s'avère incapable d'officier par lui-même.

est sans compter sur l'appétit des princes, tout aussi légitimes dans une régence pensée comme collégiale depuis l'ordonnance de $1407^{14}$. Pour s'ériger seules en chef délégué - de l'État, les reines mères doivent se battre, et justifier leur place, même si elles partent toujours largement favorites. Et cela d'autant plus que le pouvoir des femmes reste un sujet délicat dans le cadre institutionnel français. Leur exclusion de l'héritage n'a pas donné lieu, au XIV siècle, à des discours fondés sur leur inaptitude à exercer des fonctions politiques. Mais la réaffirmation de la loi salique, menacée par les visées espagnoles de la fin du XVI ${ }^{e}$ siècle, s'est portée cette fois sur le terrain de l'incapacité naturelle du sexe faible à diriger un royaume. Si l'argumentaire répondait plus à des impératifs politiques (faire de ces règles successorales des lois de nature et donc des lois divines qui ne peuvent être transgressées) qu'à des convictions anthropologiques ou biologiques, les discours s'accordaient mal avec la réalité d'une régence féminine. Le souvenir, pourtant, n'était pas si lointain, Catherine de Médicis ayant été fort présente sur la scène du pouvoir.

10 Les jurisconsultes n'étaient pas à une contradiction près mais, pour que l'édifice monarchique paraisse tenir debout, il ne fallait pas que le paradoxe soit trop manifeste. Insister sur les qualités royales de la régente (par son titre, par sa naissance aussi) et développer son caractère maternel offrait aux propagandistes de la monarchie une solution acceptable. C'est donc moins la femme (pourtant hautement qualifiée à ce titre) que la mère et la reine qui sont célébrées dans les discours sur la régence. Ces thèmes ont également le mérite de replacer la régence dans le cadre d'une loi naturelle et d'un renforcement dynastique, comme ils permettent aussi de justifier, par les liens du sang, l'extraordinaire pouvoir dont disposent les reines mères lors d'une minorité royale.

La régence féminine, entre loi naturelle et renforcement dynastique

11 Il est frappant de constater que les textes destinés à justifier la loi salique en soulignant l'inaptitude innée des femmes à exercer des responsabilités politiques évitent, pour la plupart, d'aborder la question de la régence. Et, lorsqu'ils s'attèlent à la tâche délicate qui consiste à concilier imbécillité naturelle et exercice du pouvoir - le plus souvent dans des ouvrages qui traitent de la monarchie dans son ensemble -, les auteurs cachent difficilement leur embarras. Ainsi Cardin Le Bret reconnaît, du bout de la plume, « que l'on observe encore à présent (quand les Reynes ont donné témoignage de 
leur vertu et de leurs mérites) de leur commettre la Régence et la Tutelle de leurs Enfans, jusqu'à ce que l'aîné ait atteint la quatorzieme année de son aage ${ }^{15}$. La parenthèse suffit à expliquer cette anomalie, si contraire à « la loy de la nature, laquelle ayant créé la femme imparfaite, faible et débile, tant du corps que de l'esprit, l'a soubmise sous la puissance de l'homme $»^{16}$. Les textes relatifs à la loi salique rédigés au tournant des XVI $\mathrm{XVII}^{\mathrm{e}}$ siècles ne se focalisent pas tous sur l'incapacité physique des femmes mais, si le thème est largement présent à cette époque, c'est parce qu'il confère à la démonstration une force considérable en l'inscrivant dans un ordre immuable, celui de la nature, c'est-à-dire celui de Dieu. Ce même raisonnement est poursuivi dans l'argumentaire concernant la régence, avec le thème de la nature maternelle de la reine. C'est à la fois une façon de contourner la difficulté créée par les justifications de la loi salique, et une manière de renforcer les droits de la reine mère au gouvernement du royaume pendant une minorité royale, redonnant ainsi à l'institution monarchique une cohérence que l'exclusion des femmes du trône semblait alors mettre en péril.

En fait, loi salique et régence féminine n'ont jamais fait mauvais ménage ailleurs que dans les textes relatifs aux règles successorales, et nombre de propagandistes s'accordent à reconnaitre que la seconde est une conséquence de la première. Pour Bertier de Montrave, « la loy qui fait les regens [est] une partie de celle qui fait les Roys. [...] Sans elle, la Loy Salique seroit defectueuse et imparfaitte ${ }^{17}$. Un texte anonyme rappelle que "des Reynes ont esté ordinairement préférées en ce choix à tous les princes du sang et autres seigneurs Ecclesiastiques ou séculiers, par cette mesme raison l'on ne peut pas craindre qu'elles usurpent la couronne de la succession de laquelle elles sont incapables par la loy fondamentale de ceste monarchie $\aleph^{18}$. Dupuy s'accorde à identifier parmi « les principales et essentielles considérations » de la nomination des reines mères le fait que « ces Dames, par la qualité de leur sexe, sont moins capables d'envahir l'État de leurs Enfans, que toutes autres personnes $»^{19}$. Il s'oppose ce faisant à l'opinion commune qui inscrit prioritairement « l'affection naturelle des mères envers leurs enfants " dans les raisons de ce choix, sans toutefois contester la valeur de cet argument. Car les deux sont liés, tant dans les justifications que dans l'effectivité du pouvoir des régentes. Les textes en effet n'omettent jamais de signaler l'importance du caractère maternel de la reine, y compris ceux qui associent exclusion de l'héritage et nomination à la régence. Les uns appellent cette qualité naturelle en renfort: « d'ailleurs la nature leur donne un droit inviolable à la tutelle de leurs enfants et à la conduitte de leurs jeunes frères " nous dit la suite du Recueil des Régences cité ci-dessus et qui met en avant l'exhérédation féminine. Les autres en font un élément premier, tel Savaron : car lorsque les Rois « sont en bas age, leur mère est régente, leur ayeule, leur sœur parente, portées naturellement au bien et à la grandeur de leur mineur et de l'État, d'ailleurs exemptes de passion, et de soupçon d'affecter la Royauté de laquelle elles sont forcloses $»^{20}$. Certains se contentent de souligner qu'il n'y a pas de contradiction, la loi salique maintenant des princes français sur le trône, mais « l'on ne perd point l'estime de l'autre sexe ${ }^{21}$ pour autant. L'essentiel des écrits, cependant, fait de la maternité de la reine l'argument décisif, voire exclusif, de sa régence. Du Ruau parle à ce propos de "loix puisées du sein de la Nature même " ${ }^{22}$, considérant que " la Nature seroit seule suffisante pour plaider la cause des mères ». Savaron y insiste : "l'affection d'aimer les enfants est une loy de nature escripte au cœur de toutes les mères $»^{23}$. Les lettres de nomination à la régence ne se font d'ailleurs pas faute de justifier ce choix de la sorte ; à la mère va naturellement le soin de sa progéniture. 
13 Il ne s'agit pas ici de nier la dimension anthropologique du problème. Les monarques, comme leurs contemporains, voient dans la maternité une réelle garantie pour la protection de l'enfant. Charles $\mathrm{V}$ explique ainsi le rôle qu'il accorde à la reine mère :

Les enfans meneurs d'aage doivent estre nourris et gardez par gens qui parfaictement les aiment, et esquels ne puisse ou doie avoir presomption ou soupeçon d'aucun peril ou dommage de leurs personnes, et que selon raison la mere aime d'amour piteuse plus ses enfants, et a le cueur plus doux et plus tendre d'eux nourrir amoureusement et garder soigneusement leurs corps et leurs biens, que quelconque autre personne, tant leur soit prochaine de lignage, et quant a la tutele et garde de leurs personnes doit estre preferee a tous autres ${ }^{24}$.

Près de trois siècles plus tard, Louis XIII désignait Anne d'Autriche au gouvernement des affaires pour les mêmes raisons :

la régence du Royaume, l'instruction et l'éducation des rois mineurs, ne pouvoit estre déposée plus avantageusement, qu'en la personne des mères des Roys, qui sont sans doute plus interessées en la conservation de leurs personnes et de leur Couronne, qu'aucun autre qui y pourroit estre appellé25.

15 Ce recours systématique à l'argument du droit de nature, d'autant plus performant qu'il est conforme à l'ordre social, est la réponse la plus efficace aux interdits formulés par la loi salique nouvellement justifiée. Moins que la qualité féminine, pourtant tout aussi porteuse, c'est la dimension maternelle de la mère qui est mise en avant, faisant du lien filial une valeur sûre face à la débilité naturelle dont sont accusées les femmes au même moment. Les motivations de la régente sont contenues dans ce sentiment puissant qui l'attache à son fils. Et s'il est un reproche à leur faire, c'est de «les blasmées d'exces, et non de defaut d'amour, pour les trop et non peu les aymer $\aleph^{26}$. La puissance maternelle doit être comprise comme un argument rhétorique du pouvoir, quelle que soit sa réalité par ailleurs. Anne d'Autriche ne se fait pas faute de le mobiliser lorsqu'elle "dit que le droit de nature lui a donné cet honneur que d'être régente du roi son fils, et de son royaume pendant sa minorité » et qu'à ce titre elle devrait disposer d'une autorité sans entrave ${ }^{27}$. Il est vrai que l'amour maternel s'apparente d'autant plus à une loi naturelle qu'il participe d'un ordre social traditionnel. À la mère revient régulièrement la garde des enfants, en cas de viduité, cette activité relevant, finalement, des charges domestiques dont elles sont responsables. Charles $\mathrm{V}$ confie la tutelle du roi mineur à la reine en se fondant sur cet état de fait, comme il établit la majorité royale à 14 ans en se fondant sur la tradition ${ }^{28}$. Le droit commun vient ainsi à l'appui du droit public pour justifier le pouvoir des femmes malgré l'exclusion de l'héritage. «Ladite régence est légitime par droict commun, à la mère [...] appartient la tutelle et administration des personnes et des biens de son enfant pupille, excluds tous les collateraux ${ }^{29}$. Et Bertier de Montrave de préciser : "quoy que originairement les fiefs ne pussent être tenus que par les mâles, on n'ostoit pas aux mères la garde noble de leurs enfants ${ }^{30}$.

16 Conforme à un modèle social, la régence se voit dotée de qualités féminines, les panégyriques se faisant fort de souligner la douceur d'un tel gouvernement. Mais audelà des habituelles flagorneries se retrouvent un certain nombre de thèmes propres au personnage royal féminin: la paix, la charge domestique, la maternité ${ }^{31}$. La complémentarité roi/reine œuvre encore dans le prolongement d'un couple désuni par la mort et fait de la régence, paradoxalement, une activité domestique. La dénonciation de Régnault d'Orléans - partisan parmi tant d'autres d'une débilité féminine naturelle pour valider la loi salique - se retourne au bénéfice de celle qu'il stipendie : «Voyla 
pourquoy l'homme qui a bien cogneu la naturelle inclination de la femme, luy a baillé la principauté de la maison seulement, et luy a laissé le gouvernement des choses par luy acquises $\|^{32}$. Restée seule, la reine gouverne, en nom et place du roi. La principauté de la maison, désormais élargie au royaume par l'accession au trône d'un enfant dont elle a la tutelle, reste dans la main des femmes. Mais, par voie de conséquence, le gouvernement se résume à la gestion des affaires courantes. C'est la mission dévolue à la régente, c'est le bilan dont elle s'enorgueillit. «Dieu m'a fait la grace de conserver votre Royaume et de le remettre entre vos mains au mesme estat qu'il en estoit sorti de celles du Roy monseigneur » affirme Marie de Médicis à Louis XIII pour se justifier ${ }^{33}$. Lien d'un roi à l'autre par l'enfantement, la reine assure la transition d'un règne à l'autre par sa régence. Dans cette perspective, les minorités royales s'apparentent à des périodes d'attente, sorte de parenthèses dans l'histoire du pouvoir royal, confiées à celles dont le rôle est précisément de garantir la continuité dynastique.

La mobilisation des qualités royales de la régente, acquises dès sa naissance et confirmées par son mariage, va dans le même sens. Elle permet non seulement que l'État soit dirigé par une personne de dignité supérieure - et prédisposée à ce rôle -, mais aussi de reconstituer, dans les figures du pouvoir, le couple royal, désormais composé de la mère et du fils quand il l'était autrefois du souverain et de son épouse ${ }^{34}$. Maternelle et royale, la reine bénéficie de la sorte d'une double caution qui lui assure, à elle comme au roi, l'amour de son peuple. "Quand mesme la régence est tenue par une personne illustre et sacrée, de qui tous les ancêtres ont porté couronne, chacun l'a révéré conjointement avec le Roy ${ }^{35}$. La mère et le fils réunis en une seule entité figurent à nouveau un couple régnant. Cette fois pourtant, c'est le fils qui procède de la mère, et non plus une reine définie par son époux. De fait, "Son fils est un autre ellemesme $»^{36}$, affirment certains; ce fils qui «est appelé par la loy une partie de ses entrailles ${ }^{37}$. La continuité est assurée, charnellement, par une reine qui donne la vie au roi, un roi par ailleurs successeur de son père. Héritier des qualités intrinsèques de celle qui l'a mis au monde, le jeune monarque est également formé par elle, érigée en modèle en l'absence du père. "C'est d'elle qu'il prendra des instructions qui fortifieront son excellent naturel et le rendront capable des plus grandes entreprises " prédit-on à propos de Louis XIV, monté sur le trône à l'âge de quatre ans ${ }^{38}$.

Le devoir de maternité n'est pas un vain mot : la venue d'un enfant est la seule véritable garantie, pour la reine, d'une incorporation totale et définitive à la monarchie. Bien que le mariage soit, en principe, indissoluble, et la dignité royale acquise dès les noces, la princesse est toujours, pour des raisons dynastiques évidentes, susceptible d'être remplacée en cas de stérilité. Les exemples sont rares, mais significatifs ${ }^{39}$. La régence peut ainsi apparaître comme l'apothéose d'une carrière politique qui s'amorce avec les noces et s'affirme avec un dauphin, car l'assurance d'un héritier est la clé d'un mariage réussi. En effet, l'arrivée d'un fils est essentielle à la continuité dynastique, mais également à la transmission des biens, maternels et paternels, qui ne peuvent être réunis que par héritage (le régime matrimonial royal est celui de la non-communauté des biens). L'alliance de deux familles se concrétise dans la descendance qui recueille le patrimoine. Mentionner les origines illustres des épouses et mères de roi participe au processus de glorification de la fonction royale et conforte la stabilité politique, tant par l'exercice légitime d'un gouvernement que par les prédispositions de l'héritier à gouverner à son tour. C'est une "Royne aussi grande de courage que de sang et de naissance, d'authorité en sa régence $»^{40}$ qui préside désormais aux destinées de la France. De telles affirmations augurent bien de l'avenir du royaume, tant pour 
l'exercice du gouvernement que pour les prédispositions de l'héritier du trône. La reine apporte ainsi sa part à la puissance royale, qu'elle consolide encore par l'exercice de la régence lors d'une défaillance de l'autorité masculine.

Le jeu de la filiation mérite d'être souligné. Il répond aux impératifs de la monarchie de garantir la stabilité dynastique et correspond à la mission première, et principale, assignée à la reine. C'est parce qu'elle devient mère qu'elle obtient la régence, c'est parce qu'elle est elle-même dans la continuité d'une lignée prestigieuse qu'elle est en mesure de diriger les affaires de l'État, "ayant une meilleure naissance, et une nourriture plus relevée que le vulgaire, l'on en pouvoit attendre que des actions héroiques $»^{41}$. La construction familiale, c'est-à-dire dynastique, passe par un double apport - féminin et masculin - qui explique, sinon justifie, le rôle clé de la mère dans toutes les modalités de la transmission. La maternité en est le vecteur, elle préfigure la puissance féminine. Le modèle social rejoint une fois de plus les dispositions d'ordre institutionnel.

La reine mère au pouvoir : pouvoir des femmes, pouvoir des mères

20 Au-delà d'une simple légitimation de la place qui est désormais sienne à la tête de l'État, la maternité confère à la reine un pouvoir considérable qui tient autant aux dispositions institutionnelles qu'à l'ascendant qu'elle est en mesure d'exercer sur son fils. La mission confiée à la souveraine en période de minorité royale est à priori aussi claire qu'entravée : suivre les directives proposées par les conseillers que le roi désigne aux côtés de sa femme afin de maintenir le royaume en l'état jusqu'à la majorité de l'héritier. Dans les faits, de telles prérogatives se traduisent par l'exercice d'un pouvoir qui n'est limité que dans le temps. Et encore; la majorité, à quatorze ans, du jeune monarque met rarement fin à l'influence d'une mère qui troque alors son titre de régente pour celui de surintendante de l'État, à la demande de son fils ${ }^{42}$. Gouvernant au nom du roi, la reine est censée ne prendre aucune initiative qui puisse remettre en cause la politique menée jusque-là. Mais pour ce faire, elle est déclarée régente « avec toute puissance et autorité $\aleph^{43}$. Il y a là une contradiction dont la reine mère sait habilement jouer pour éliminer tout pouvoir concurrent. Le raisonnement mené par Anne d'Autriche pour obtenir les pleins pouvoirs est significatif du parti à tirer de cette situation. Le 18 mai 1643, au lendemain de la mort de Louis XIII, la toute récente veuve amène Louis XIV tenir un lit de justice, et déclare :

Qu'elle avoit amené le Roy en son Parlement, pour dire, que bien que le feu Roy [...] l'eust declarée Régente de sa personne et de son Royaume pendant sa minorité, et luy eust donné des Ministres qu'elle ne pourroit destituer, et sans lesquels elle ne pourroit disposer des affaires importantes de son Estat: Neantmoins, parce que cette Declaration estoit contraire aux loix fondamentales du Royaume, et qu'elle bornoit le pouvoir absolu qu'elle devoit avoir en qualité de Régente, elle entendoit avoir une autorité libre et absoluë; et pour cet effect avoit fait assembler la Compagnie ${ }^{44}$.

21 À sa suite, Omer Talon, premier avocat du Roy, renchérit :

La reine, laquelle ayant la régence de son fils par le droit de la nature, et par le choix qu'en avoit fait le Roy defunt son mary, il estoit à propos qu'elle eust la liberté de choisir tel Conseil, et tels Ministres qu'il luy plairoit, sans estre astrainte à ceux qui estoient denommez en la Declaration du feu Roy; lesdits ministres devant estre plustost par son election, que par necessité, veu l'authorité Royale et monarchique qui n'a pû estre partagée ${ }^{45}$.

L'administration entière des affaires du roi mineur ne soufre pas d'entraves dans son exercice. Bien des jurisconsultes insistent sur ce point : la régence des reines mères doit 
être absolue car «elles tiennent leur autorité directement de la loy de l'Estat $»^{46}$. L'incapacité des femmes à régner par elles-mêmes favorisent une telle position de force, que les lois naturelles d'un amour maternel accréditent encore. Le duc d'Orléans, premier prince du sang et à ce titre héritier du trône juste après le roi ne dispose pas absolument de cette marge de manœuvre. Car « il s'en faut bien [...] qu'un prince du sang soit au Roy ce que doit luy estre sa mere ${ }^{47}$. L'argument du lien maternel mère/ fils joue dans les deux sens : à l'amour maternel qui garantit les intérêts du roi mineur et autorise les pleins pouvoirs répond l'amour filial qui enracine plus profondément encore l'autorité de la reine. Le soutien du roi est fondamental : c'est en son nom que s'exerce le pouvoir ; c'est lui qui retient, en aval, toute l'autorité. Pour autant, il reste durablement sous l'influence de celle qui marque son enfance. Elle l'instruit et, par sa présence constante, lui apprend le métier de roi, jouant à elle seule le rôle des deux parents en l'absence du père ${ }^{48}$. Cette situation n'est pas sans conséquence sur l'attitude du roi mineur à l'égard de sa mère, et explique les tensions qui peuvent surgir alors au sein de la cour. « Il y en a [...] qui sont jaloux de l'honneur et du respect que le Roy rend à la Reyne, naturellement portée à faire régner son fils $»^{49}$. Outre le sentiment royal exprimé ici, la rhétorique est intéressante en ce qu'elle retourne l'ordre des propositions : ce n'est plus tant le roi qui délègue à la régente l'autorité nécessaire à la direction des affaires de l'État, mais la mère qui « fait régner son fils » et cautionne de ce fait sa souveraineté.

Le couple mère/fils constamment mobilisé dans les représentations de la régence féminine ${ }^{50}$ est essentiel à l'image d'une reine qui se présente comme l'instrument de la volonté du prince, le bras qui soutient l'édifice monarchique. Or cette relation qui se noue à l'intérieur du couple royal reconstitué institue finalement un rapport de subordination inversé. Éducatrice, protectrice, la reine a autorité sur la source même de son autorité. Louis XIII n'a guère plus de neuf ans lorsqu'il accède au trône et « dans cette foiblesse, il avoit plus besoin d'estre gouverné luy-mesme, que de gouverner les autres, la tutelle du Roy et la Regence de l'Estat est deferée à la Reyne sa mere " ${ }^{51}$. Le poids de la régente se fait donc sentir sur l'ensemble du royaume, y compris sur le roi lui-même, incapable de se soustraire véritablement à l'emprise de celle qui l'initie aux affaires de ce monde. La puissance d'Anne de Beaujeu à la tête de l'État passe par celle qu'elle exerce sur Charles VIII : «Elle ne voulut en rien du tout relascher de l'autorité qu'elle avoit usurpée en l'administration des affaires d'Estat, sous ombre du Gouvernement de la personne du Roy qui luy avoit esté confiée, et qui dans un âge de plus de quinze ans, ne parloit encore que par la voix de sa Gouvernante, et n'agissoit que par son organe $\aleph^{52}$. Les gouvernements du roi et du royaume se confondent dans l'action. Il y a là un reproche fréquent adressé au pouvoir féminin. Anne de Beaujeu, encore, est accusée par Louis d'Orléans de "tenir le Roy en subjection " ${ }^{33}$, double crime qui consiste à accaparer le pouvoir et à renverser l'ordre naturel de distribution de l'autorité. Enfin, si les frondeurs cherchent à gagner le roi, c'est pour le soustraire à l'influence déplorable d'Anne d'Autriche et Mazarin... et discréditer la régente.

La charge éducative favorise le contrôle spirituel du jeune roi mais lorsque, au devoir de reconnaissance, s'ajoute l'amour filial, la domination est totale : "il est certain que Louyse de Savoye eut tousjours grande part au Gouvernement des affaires publiques durant tout le regne de son fils, lequel avoit une confiance tres-particulière en sa bonne conduite, et conserva tousjours envers elle durant toute sa vie, un amour cordial, et un respect inviolable $»^{54}$. Catherine de Médicis sait à son tour user des sentiments qu'elle 
inspire à ses fils. Malgré les données institutionnelles, «elle eust tousjours esté Regente, mesme durant qu'ils estoient Roys majeurs, et absolus sur leurs peuples ${ }^{55}$. Et le même texte de renchérir : "Enfin par le respect particulier que le Roy Henry III conserva tousjours pour sa Mere, elle eut autant de part à toutes les affaires durant son regne, comme si elle eust esté encore Regente $»^{56}$. La force de la filiation est d'ailleurs un atout que Catherine mobilise à tout instant. Sa correspondance rappelle, chaque fois qu'il est question du souverain, qu'il s'agit bien $\mathrm{du}$ « roi mon fils », soulignant ce faisant qu'elle n'intervient dans les affaires d'État qu'au titre de reine mère, titre qu'elle conserve jusqu'à sa mort, bien au-delà du terme légal de sa régence ${ }^{57}$.

Amour et devoirs filiaux, confusément mêlés, imposent reconnaissance, affection, attachement, sous peine d'apparaitre comme un fils indigne. Convenances sociales ou sentiments vrais, la gratitude est de règle jusque dans le bilan que les rois dressent de leur minorité. Un traité des régences, rédigé probablement dans la première moitié du XVIII siècle, sous le gouvernement de Philippe d'Orléans, constate qu'effectivement il n'y eut guère de condamnation, par les monarques, de l'œuvre des reines mères. L'auteur en donne l'explication : « les Reynes ont toujours eté Regentes depuis Charles VI. Il est simple de penser que le respect de leurs enfans ne leur a pas permis de chercher a critiquer leur conduite $»^{58}$. Force est de constater que les plaintes sont restées l'apanage des particuliers. Pour les reines mères, la postérité est assurée, au moins officiellement, en vertu d'un lien qui n'autorise pas le désaveu.

Pour autant, les relations mères-fils peuvent être conflictuelles, et la gratitude n'est pas forcément dénuée de craintes envers celle qui cumule les charges de père et de mère. L'attitude de Louis XIII envers Marie de Médicis, fermement décidée à se maintenir au pouvoir en faisant pression sur l'adolescent, est révélatrice des difficultés que peut rencontrer un jeune prince pour s'affirmer face à sa tutrice. Incapable d'affronter sa mère directement, Louis XIII s'en prend aux favoris, Concini et Léonora Galigaï, qu'il fait assassiner, avant de placer la reine douairière en résidence surveillée. Le récit que nous offre un texte pourtant destiné à réhabiliter cette mère qui se veut éplorée en dit long sur l'ambiguïté des sentiments qu'échangent mère et fils :

Quant à la Royne mere du Roy estant arrestée dans sa chambre sa Majesté, luy fit incontinent scavoir, qu'elle estoit desormais resolue, de scavoir ses affaires et prendre cognoissance de ce qui se passe en son Estat, désiroit qu'elle ne s'en meslast plus et qu'elle luy en laissast le soing. Qu'elle la prioit de trouver bon de se retirer, qu'elle donneroit ordre à son partement, et au surplus qu'elle s'asseurast que sa Majesté l'honoreroit tousjours comme sa mere. [Marie de Méd. demande alors une entrevue avec son fils avant de partir] Le Conseil d'autre part, bien que sa Majesté eust pris une résolution inesbranlable et s'y fust fortifiée par le hazard qu'elle avoit courru, et qu'elle n'apréhendast nullement les parolles, ny les gestes de sa mere, toutefois les charmes de la nature sont tels qu'il est presque impossible aux hommes d'y resister, a plus forte raison à ceux qui n'ont encor atteint cette force entiere, aussi que tous les ordres de l'Estat en general, et chacun en particulier ne désiroit plus vivre sous la loy d'une femme dont aussi tous les jours y avoit plainte à sa Majesté. Cela fut cause qu'il fut arresté que sa Majesté verroit ladite Dame en presence de Messieurs de son Conseil ${ }^{59}$.

Le roi, malgré la détention entière et sans entrave de sa souveraineté, et de la pleine possession de ses moyens depuis sa majorité, ne s'estime pas la force de résister à celle dont on lui rappelle ailleurs qu'elle « vous a fait Roy, en conservant vostre vie dans son ventre, et vostre couronne durant sa régence $»^{60}$. L'attitude timorée de Louis XIII dans un contexte de conflit ouvert confirme les analyses conduites sous les régences : tenir 
le roi, c'est tenir l'État ${ }^{61}$, et l'ascendant d'une reine mère dépasse largement le cadre circonscrit de son autorité déléguée.

Sans contester la validité d'un amour maternel dont il est difficile de vérifier l'exactitude, il est évident que la reine en fait par ailleurs un instrument de son pouvoir. Les lits de majorité, qui marquent officiellement la fin d'une régence, servent en réalité à « continuer l'autorité dans le gouvernement de l'État ${ }^{62}$, juge Dupuy avec discernement. Forte des exemples précédents qui ont vu, lors de cette cérémonie, la confirmation du pouvoir des régents, Anne d'Autriche organise à son tour une restitution de son autorité dans un lit de majorité dont la mise en scène porte sur une répartition équivoque de la soumission. Dans un projet dressé pour les cérémonies de cet acte, il est dit : «la reine sortira de sa séance comme voulant rendre hommage au Roi, lequel descendant de son lit de justice la vient embrasser ${ }^{63}$. Comptant sur les sentiments de son enfant, la reine n'hésite pas à présenter l'image d'une parfaite sujétion, se plaçant ainsi sous l'entière puissance d'un roi désormais majeur, mais encore trop jeune pour s'émanciper totalement de l'influence maternelle. La manœuvre réussit, sans que le succès ne soit le résultat d'un risque considérable. L'enfant royal se lève pour embrasser sa mère. Le baiser qu'Anne d'Autriche rend aussitôt témoigne d'une relation qui dépasse le registre du pouvoir pour s'inscrire dans une autre catégorie, celle d'une profonde affection. Cependant, c'est bien de pouvoir qu'il s'agit in fine, pouvoir de la reine sur le roi, de la régente dans l'État. La suite le confirme : «je vous prie de continuer à me donner vos bons avis. Je désire qu'après moi vous soyez le chef dans mon conseil » se voit répondre Anne d'Autriche par Louis XIV ${ }^{64}$. Belle victoire d'une femme sur un pouvoir réputé masculin, la régence est aussi le triomphe de la maternité.

\section{BIBLIOGRAPHIE}

AMIABLE Louis, 1861, «Essai historique et critique sur l'âge de la majorité », Revue d'Histoire du droit français et étranger, t. 7 .

BERTIER DE MONTRAVE, 1649, La regence a l'entrée du Parlement de Tolose, Toulouse, J. Boude.

BLUCHE François, 1986, Louis XIV, Paris, Fayard.

CORVISIER André, 2002, Les régences en Europe, Paris, PUF.

COSANDEY Fanny, 2004, « Représenter une reine de France. Marie de Médicis et le cycle de Rubens au palais du Luxembourg », CLIO, Histoire, Femmes et Sociétés, 19, p. 63-83.

COSANDEY Fanny et DESCIMON Robert, 2002, L'absolutisme en France. Histoire et historiographie, Paris, Le Seuil.

COSANDEY Fanny, 2000, La reine de France, symbole et pouvoir, Paris, Gallimard.

COSANDEY Fanny, 2002, « Francese o Straniera ? La regina di Francia fra dignità regale e successione ereditaria », Genesis, Rivista della Società Italiana delle Storiche, 1/1, p. 35-60. 
Declaration du roy sur la Régence de la Reyne, vérifiée en Parlement le 21 avril 1643, Paris, A . Estienne et P. Recolet.

DES MORGUES Mathieu, 1631, La tres humble, tres veritable, et tres importante remonstrance au Roy, imprimée l'an 1631, dans : Diverses pieces pour la defense de la royne mere du roy, s.l.

DES MORGUES Mathieu, 1637, Diverses pièces pour la défense de la Royne mère du Roy tres Chrestien Louis XIII, s.l.

Discours sur ce qu'aucuns seditieux ont temerairement dit et soutenu que pendant la minorité des Rois de France, leurs meres ne sont capables de la regence dudict Royaume, ains qu'elle appartient seulement aux Princes masles qui sont plus proches et habiles a succeder a la couronne, 1579, Paris, N. Roffet.

Discours sur les regens qui ont gouverné l'Estat sous les Roys de la troisieme race depuis l'an 987, BNF, Ms NAF 2080.

DOLAN Claire, 1998, Le notaire, la famille et la ville (Aix-en-Provence à la fin du XVI ${ }^{e}$ siècle), Toulouse, Presses Universitaires du Mirail.

D'ORLEANS Regnault, 1597, Les observations de diverses choses remarquees sur l'Estat, couronne et peuple de France, s.l.

Du conseil des Roys, et du gouvernement de leur Estat. Avec quelques observations sur la régence, Bibliothèque Nationale, Fonds Morel de Thoisy, t. CIII, fol. 1, s.l.n.d. (v. 1643).

DU CREST Aurélie, 2002, Modèle familial et pouvoir monarchique, Aix-en-Provence, Presses Universitaires d'Aix-Marseille.

DU RUAU Florentin, 1615, Le Tableau de la Régence de Blanche Marie de Médicis, Royne mere du Roy et du Royaume, Poitiers, A. Mesnier.

DU TILLET Jean, 1570, De l'Estat et succez des affaires de France, Paris.

DUPUY Pierre, 1655, Traité de la majorité de nos rois et des regences du Royaume avec les preuves [...] Ensemble un traité des preeminences du parlement de Paris, Paris, veuve Mathurin Dupuy et Edme Martin.

GODINEAU Dominique, 2003, Les femmes dans la société française XVI ${ }^{e}$-XVIII ${ }^{e}$ siècle, Paris, Armand Colin.

HANLEY Sarah, 1989, « Engendering the State : Family Formation and State Building in Early Modern France », French Historical Studies, 16/1, p. 4-27.

LA FERRIERE Hector de, BAGUENAULT de PUCHESSE Gustave, A. LESORT, 1880-1943, Lettres de Catherine de Médicis, Paris, Documents inédits de l'histoire de France, 12 vol.

La régence des Reynes en France, ou les régentes, s.l.n.d.

LE BRET Cardin, 1632, De la souveraineté du Roy, Paris, Jacques Quesnoy.

Les humbles supplications de la Royne mere du Roy, 1619, Paris, s.l.

Majorité de Louis XIV, 1651, A.N. KK 1438-2, fol. 343 vº.

MERLIN Hélène, 1994, Public et littérature en France au XVII ${ }^{e}$ siècle, Paris, Les Belles Lettres.

Recueil des regences qui ont esté en France depuis Pharamond jusque à present avec quelques choses particulieres arrivees soubs ces regnes là, sd, BNF, Ms Fr. 9621.

SAVARON jean, s.d, Traicté de la régence, à la reine régente (v. 1615), BNF, Ms Fr. 17299.

Traité des regences, BNF, Ms Fr. 10796 (XVIII ${ }^{\mathrm{e}}$ siècle). 
TURQUET DE MAYERNE Louis, 1611, La monarchie aristodemocratique, Paris, I. Borjon.

\section{NOTES}

1. Cosandey $2000: 3^{\mathrm{e}}$ partie chapitre 2 .

2. Cf. Corvisier 2002.

3. Cf. Merlin 1994, sur cette notion du "public".

4. Cf Godineau 2003, mais aussi Dolan 1998 par exemple qui souligne le rôle des femmes, en particulier des veuves, dans la société aixoise.

5. La dénonciation du gouvernement des reines mères participe d'une littérature d'opposition, et doit être considérée comme des attaques conjoncturelles bien plus que comme l'expression d'un dysfonctionnement structurel de la monarchie. Certains auteurs cependant - mais ils sont rares - n'acceptent aucune forme de domination féminine. C'est le cas, par exemple, de Turquet de Mayerne 1611.

6. Hanley1989.

7. Du Crest 2002.

8. Cosandey et Descimon 2002.

9. Cosandey 2002.

10. Pour un développement de la question, Cosandey 2000, dont sont tirées les conclusions exposées ici.

11. Voir Dupuy 1655 : 18.

12. Elle est aussi la mère de la princesse Claude de France, fiancée au futur François I $^{\mathrm{er}}$.

13. Il s'agit de Louise de Savoie.

14. Si cette figure de la régence, exercée en commun par la reine, les princes et les principaux ministres, n'est guère mise en pratique, elle reste un modèle à suivre par les rois qui, dès que l'occasion se présente, rédigent leur testament en ce sens.

15. Le Bret $1632: 45$.

16. Le Bret 1632 : 31 .

17. La régence à l'entrée du Parlement de Tolose $1649: 5$.

18. Recueil des régences qui ont esté en France... s.d. : 189.

19. Dupuy $1655: 35$.

20. Savaron (v. 1615) : fol. $115 \mathrm{v}^{\circ}$.

21. Du conseil des Roys... sd : 11 .

22. Du Ruau 1615 : 10.

23. Savaron (v. 1615) : fol. 121.

24. Cité par Dupuy 1655, Preuves du traité : 169.

25. Declaration du roy sur la Régence de la Reyne... 1643.

26. Savaron (v. 1615) : fol. 121.

27. Séance du lit de justice de Louis XIV, le 18 mai 1643. Discours du chancelier, rapporté par Dupuy 1655, Preuves du traité : 532.

28. Amiable $1861: 228$.

29. Discours sur ce qu'aucuns séditieux... $1579: 5$.

30. Bertier de montrave $1649: 9$.

31. Voir à ce propos les thèmes développés dans les entrées royales, Cosandey $2000: 2^{\mathrm{e}}$ partie, chapitre II.

32. Régnault d'Orléans $1597: 72$.

33. Lettre rapportée par Des Morgues $1637: 613$. 
34. Cosandey 2004.

35. Du conseil des Roys... v. $1643: 8$.

36. Discours sur les regens : 102.

37. Bertier de Montrave $1649: 7$

38. Du conseil des rois... v. $1643: 9$.

39. Ainsi Jeanne de France, première épouse de Louis XII, et Marguerite de Valois, première femme de Henri IV, toutes deux sans descendance légitime, ont vu leur mariage annulé. Catherine de Médicis, longue à être mère, s'est trouvée dans les premiers temps de son mariage dans une situation très fragile de ce fait.

40. Du Ruau $1615: 67$.

41. Du Ruau $1615: 67$.

42. Cosandey 2000.

43. Savaron 1615 : fol. $115 \mathrm{v}^{\circ}$.

44. Dupuy 1655, Preuves du traité : 532.

45. Dupuy 1655, Preuves du traité : 533.

46. Bertier de Montrave $1649: 8$.

47. Traité des régences (XVIIIe siècle) : 373.

48. Intervient ici une dimension psychanalytique du problème qu'il serait intéressant de traiter.

49. Du Ruau 1615 : 724.

50. Cosandey 2000.

51. La régence des Reynes en France, ou les régentes s.d. : 80.

52. La régence des Reynes en France, ou les régentes s.d. : 80.

53. Dupuy 1655, Preuves du traité : 268-69.

54. La régence des Reynes en France, ou les régentes s.d. : 63.

55. La régence des Reynes en France, ou les régentes s.d. : 73.

56. La régence des Reynes en France, ou les régentes s.d. : 76.

57. Voir la correspondance de Catherine de Médicis, La Ferrière, Baguenault de Puchesse, Lesort 1880-1943.

58. Traité des regences (XVIII ${ }^{\mathrm{e}}$ siècle) : 381-382.

59. Les humbles supplications de la Royne mere du Roy 1619 : 4-5.

60. Des Morgues $1631: 54$.

61. On dit encore d'Anne de Beaujeu qu'elle « avoit et possedoit le Roi », Du Tillet 1570 : 268-9.

62. Dupuis $1655: 120$.

63. Majorité de Louis XIV 1651 : fol. 343.

64. Cité par Bluche $1986: 76$.

\section{RÉSUMÉS}

Si la loi salique empêche les femmes de régner par elles-mêmes, elle leur confère finalement un pouvoir considérable lors des minorités royales. Incapables d'usurper, les reines mères se voient régulièrement confier le gouvernement en même temps que l'éducation de leur jeune fils à la 
mort de l'époux. L'amour maternel, inscrit dans le registre des lois naturelles, est un argument régulièrement évoqué, tant pour contrer la naturelle incapacité des femmes à gouverner que les jurisconsultes avancent pour justifier la loi salique, que pour appuyer l'autorité sans pareille des régentes. Mais au-delà d'une rhétorique du pouvoir, le lien filial qui unit mère et fils, lesquels sont aussi reine et roi, permet la reconstitution d'un couple royal qui représente à la fois continuité dynastique et stabilité politique. A ceci près que, dans cette figure du pouvoir reconstituée, le fils procède de la mère quand la reine devait son titre à l'époux. Ainsi, le devoir de maternité imposé à la souveraine permet certes un accroissement de la puissance monarchique, ne serait-ce que par la transmission, au jeune monarque, des héritages maternels et paternels, mais assure aussi à la reine de France une puissance que les bornes imposées par l'institution ne peuvent contenir, en contrôlant, par l'exercice de la régence, la source même de son autorité.

\section{INDEX}

Mots-clés : education royale, héritage maternel, loi naturelle, loi salique, minorité royale, monarchie, puissance maternelle, régence, tutelle et gouvernement, Reine de France

\section{AUTEUR}

\section{FANNY COSANDEY}

Fanny COSANDEY est maître de conférences à l'Université de Nantes. Dans la continuité de ses travaux sur La reine de France, Paris, Gallimard, 2000, elle travaille sur les questions de pouvoir royal et de puissance dynastique en France sous l'Ancien Régime. Elle a publié récemment, en collaboration avec Robert Descimon, L'absolutisme en France, histoire et historiographie, Paris, Le Seuil, 2002, et assuré la direction de l'ouvrage Dire et vivre l'ordre social en France sous l'Ancien Régime, Paris, Presses de l'EHESS, 2005. 